\title{
MICROBIAL COMMUNITY FUNCTIONAL DIVERSITY IN AN ORGANOCHLORINE CONTAMINATED SITE AND EVALUATION OF BIODEGRADATION EFFICIENCY
}

\author{
ZHU, C. ${ }^{1 \#}-$ WANG, D. ${ }^{1 \#}-$ ZhANG, J. W. ${ }^{2,4}-$ QU, C. S. ${ }^{1}-$ HuA, Z. L. ${ }^{3}-$ ZhANG, M. C. ${ }^{1}-$ WANG, S. $^{1 *}$ \\ ${ }^{1}$ Key Laboratory of Environmental Engineering, Jiangsu Provincial Academy of Environmental \\ Science, Nanjing, Jiangsu 210036, China \\ ${ }^{2}$ School of the Environment, Nanjing University, Nanjing, Jiangsu 210036, China
}

${ }^{3}$ College of Resources and Environmental Sciences, Nanjing Agricultural University, Jiangsu 210036, China

${ }^{4}$ Department of Public Health, University of Copenhagen, Copenhagen, Denmark

${ }^{\#}$ These authors have contributed equally to this article

*Corresponding author

e-mail: wangshui@jsaes.com; phone: +86-25-5852-7708; fax: +86-25-5852-7706

(Received $16^{\text {th }}$ May 2019; accepted $16^{\text {th }}$ Jul 2019)

\begin{abstract}
A typical chlorinated hydrocarbon contaminated site was selected as the research object in southern China. First, Gas Chromatography-Mass Spectrometer (GC-MS) was used to analyze the composition and concentration of pollutants in soil and groundwater samples. Then, we investigated the microbial community structure, composition, and abundance by high-throughput sequencing analysis. Principal component analysis and environmental factor correlation analysis were further used to illustrate the functional diversity of microbial communities. Eventually, the results showed that the composition and structure of the microbial community were significantly different due to the various degrees of pollution in the soil and groundwater. In the soil with high pollution degree, the functional diversity of the microbial community was notably different from that of non-polluted or slightly polluted soil. This indicated that soil microbial physiological activities and community functional diversity could be affected by the residual concentration of chlorinated hydrocarbons in contaminated soils. The dominant microbial community in the soil was Penicillium, which had a higher abundance in the soil environment, while the Phylum Firmicutes were more sensitive species. In addition, as stain-resistant bacteria, Brevundimonas, Rhizobium Frank, and Pseudomonas were observed in the groundwater, where Lasiodiplodia was the dominant microbial community, while Fusarium was more sensitive species.
\end{abstract}

Keywords: chlorinated hydrocarbon, soil and groundwater microorganism, community structure, species diversity, degradation

\section{Introduction}

Due to the rapid development of urbanization in China, local governments have promoted the strategic transformation of industrial structure and urban layout, revealing problems of abandoned industrial buildings and resources facing relocation. A large number of heavily polluting industrial enterprises have been moved into industrial parks, which were originally in the main urban areas.

Through the long-time production history, a large amount of toxic and harmful substances persist in the soil of site thanks to some heavily polluting enterprises, because of improper operations, leakages, insufficient environmental supervisions and some other reasons, adversely affecting the local ecological environment, the health of 
local people as well as land redevelopment and utilization. Organic pollutants have always been the vital monitoring targets in the investigation of contaminated sites. Most of them are generally bio-accumulative, chronically toxic and have a "three-way" (carcinogenic, teratogenic and mutagenic) effect, which have been listed as priority pollutants in the world (Su et al., 2015). At present, the soil and groundwater pollution situation in China is severe. In addition, according to reliable data, most of the contaminated sites contain chlorinated hydrocarbons and aromatic hydrocarbons (Tan et al., 2017). Chlorinated hydrocarbons have been extensively studied for their high toxicity, refractory degradation, and strong accumulation. According to the survey, once chlorinated hydrocarbons enter the groundwater, if they are not effectively repaired, the pollution can last for decades, even hundreds of years (Wang, 2018).

Chlorobenzene and chlorinated alkane are common pollutants in the soil of chemical pollution sites in Jiangsu Province. In addition to chemical detection and analysis methods, biological diagnosis is also an important means to assess the environmental risks associated with organically contaminated soils (Gao, 2014). The community structure and functional diversity of microorganisms have long been regarded as important biological indicators in soil and groundwater environments, and have received extensive attention in recent years. Through laboratory testing of microbial indicators in contaminated sites, the composition, concentration and biological toxicity of pollutants in contaminated sites can be accurately determined, thus providing a detailed and accurate reference for the risk assessment and remediation of contaminated sites. The specific indicators are as follows: (1) in the soil environment, the routine indicators of microorganisms include microbial biomass, microbial community structure, microbial functional diversity, microbial genetic diversity, total soil DNA, total soil RNA, etc.; (2) in the groundwater environment, routine microbiological indicators include, number of coliforms, heat-resistant coliforms, Escherichia coli, and total number of colonies.

Soil and groundwater microbial community diversity is very sensitive to changes in soil chemical properties and can be used as an important biological indicator to measure soil and groundwater quality and to assess the sustainability of soil ecosystems. The site pollution habitat is a special habitat composed of site pollutants-soil-groundwatermicroorganisms (Song, 2015). A series of physical and chemical changes, caused by external pollutants, will occur in the soil and groundwater, and profoundly affect the environmental and biological properties of the site. High-throughput sequencing technology can be applied to evaluate the functional diversity of microbial communities in the site (Zhang et al., 2018; Shi et al., 2018; Li et al., 2019), such as comparing different soil types (Luo et al., 2013), different plant species in the same soil (Nie, 2013), and the diverse functions of soil microbial communities in different vegetation rhizosphere (Liang et al., 2018; Qiu et al., 2019). However, studies on the functional diversity of soil and groundwater microbes in chlorinated benzene and chlorinated alkane-contaminated sites using high-throughput sequencing technology have rarely been reported.

We attempted to use high-throughput sequencing technology to analyze the soil and groundwater microbial community functions and diversity of chlorobenzene and chlorinated alkane-contaminated sites, and to study the relationship between soil and groundwater pollution levels and microbial activity. Therefore, the present study was aimed to provide details of the spatial variations of the bacterial communities in surface sediments collected from different locations within the contaminated site. 


\section{Materials and methods}

\section{Brief introduction of the sampling site}

The soil and groundwater samples selected for this study were from a decommissioning industrial contaminated site that was used as a point of production for chemical products. The enterprise was located in Taizhou, Jiangsu province, China, and focused on new material industry, and engaged in chlorine alkali, methane chloride, and fine chemicals production in 1960 to 2017.

\section{Collection and analysis of soil and groundwater samples}

The samples were collected from an industrial site of a chemical plant in May 2017. Five-point sampling methods were adopted to collect soil samples, and the hardened parts on the surface were removed. The depth of sampling for soil analysis was at $4 \mathrm{~m}$ and $7 \mathrm{~m}$ of S1 and S11, respectively. Soils were air-dried in shade and sifted through 10 meshes for analysis. The groundwater table was $0.8 \mathrm{~m}$, and depth of the groundwater monitoring well construction was $9 \mathrm{~m}$. The water-table contour map shows that the direction of groundwater flow is west to east (Fig. 1). Groundwater samplings were from Groundwater well No.11, No.1 and No. 6, as shown in Figure 1.

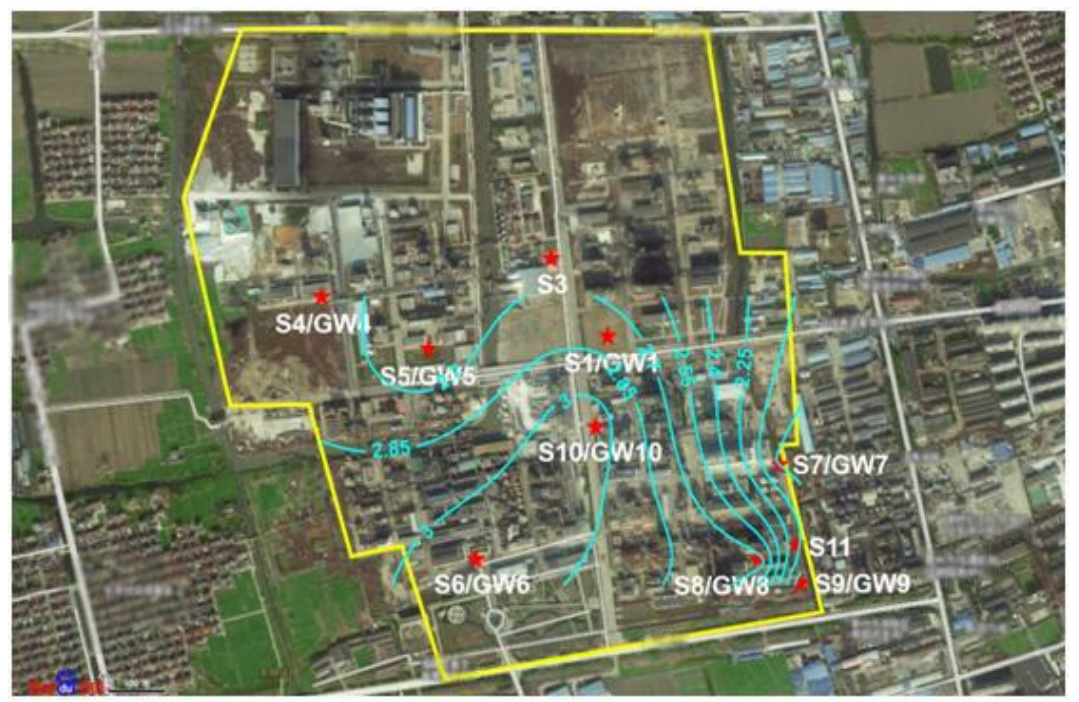

Figure 1. Groundwater flow direction of the contaminated site

After extraction, centrifugation, activation and purification, the samples were placed in Gas Chromatography-Mass Spectrometer (GC-MS) for analysis with helium as carrier gas. According to the obtained mass spectrogram, the database was searched. The result shows that the main pollutants in the environment are halogenated aliphatic compounds and chlorinated hydrocarbons.

\section{Soil contaminant}

There are about 20 kinds of organic contaminants in the soil samples, including entachlorobenzene, hexachlorobenzene, hexachloroethane, hexachlorobutadiene, phenol, phenanthrene, quinone, total petroleum hydrocarbon, dichloromethane, carbon tetrachloride, pentachloroethane,1,2-dichloroethane, 1,1,2-trichloroethane, 1,1,2,2- 
tetrachloroethane, cis-1,2-dichloroethylene, trichloroethylene, tetrachloroethylene, chlorobenzene, carbon disulfide, chloroform. The contaminant concentration of soil sample at depth $4 \mathrm{~m}$ was higher than that of $7 \mathrm{~m}$ soil sample, such as chloroform was over 10.5 times.

Compared with the "Site Soil Environmental Risk Assessment Screening Value" (DB11/T 811-2011) (industrial land), there were excessive levels of methylene chloride, carbon tetrachloride and chloroform in the soil. Their values were respectively 4.3 times, 26.6 times, and 207 times the standard.

\section{Groundwater contaminant}

About 29 organic substances were detected in groundwater, including hexachloroethane, hexachlorobutadiene, phenol, 2-methylphenol, 3-methylphenol, 4methylphenol, total petroleum hydrocarbons, 1,2-dichloride Propane, vinyl chloride, 1,1-dichloroethylene, dichloromethane, trans-1,2-dichloroethylene, cis-1,2dichloroethylene, carbon tetrachloride, 1,2-dichloroethane, trichloroethylene, 1,1,2trichloroethane, 1,3-dichloropropane, tetrachloroethylene, 1,1,2,2-tetrachloroethane, 1,2,3-trichloropropane, pentachloroethane, toluene, ethylbenzene, m-xylene and oxylene, o-xylene, carbon disulfide, chloroform. Compared with Regional Screening Level (RSL) Summary Table (TR = 1E-06, HQ = 1) April 2019. In the G3 groundwater sample, no pollutants other than the above organic substances were detected.

\section{High-throughput sequencing analysis of soil and groundwater microorganisms}

According to the manufacturer's protocols, Microbial DNA was extracted from soil and groundwater samples using the E.Z.N.A.S. tool DNA Kit (Omega Bio-tek, Norcross, GA, U.S.). The amplification of the V3-V4 region of 16S rDNA and the Internal Transcribed Space (ITS) region of Eukaryotic Ribosomal RNA gene were examined by Polymerase Chain Reaction (PCR). Purified amplicons were pooled in equimolar and paired-end sequenced $(2 \times 250)$ on an Illumina platform according to the standard protocols. Then low quality reads with less than $80 \%$ bases with quality (Qvalue) of 20 or lower and/or over $10 \%$ bases unknown $(\mathrm{N})$ were removed from each data set to gain more reliable results. After that, the clean reads of high quality from all soil and groundwater samples were merged together and assembled by using FLASH (v 1.2.11) with a minimum overlap of $10 \mathrm{bp}$ and mismatch error rates of $2 \%$. The resultant high-quality sequences were processed to generate operational taxonomic units (OTUs) by UPARSE pipeline at the $97 \%$ sequence similarity threshold. The taxonomic categorification was performed with the RDP classifier based on UNITE Database (Wu et al., 2018) (https://unite.ut.ee/) and SILVA Database (Rosselli et al., 2016) (https://www.arb-silva.de), respectively. Based on the species abundance of each sample in the OUT list, vegan packages are used in the $\mathrm{R}$ to calculate 3 types of microbial diversity index (Chao1, Shannon and Coverage), and ggplot2 packages are used in the $\mathrm{R}$ to perform the principal component analysis. Chao1 index presents the total number of OUT in the sample; Shannon index shows the degree of $\alpha$-diversity of species in the microbial community; and Coverage index presents the Database's coverage rate for different sample. 


\section{Results}

\section{Analysis of bacterial diversity in site soil and groundwater}

The contaminant concentration of GW3 sample was high. In particular, the concentration of GW1 sample, of which 1, 2-Dichloropropane, carbon tetrachloride and chloroform reached to $19.5 \mu \mathrm{g} / \mathrm{L}, 193 \mu \mathrm{g} / \mathrm{L}$ and $63 \mu \mathrm{g} / \mathrm{L}$, respectively. The $1,2-$ dichloroethane concentration of GW2 was $1.3 \mu \mathrm{g} / \mathrm{L}$. GW1 served as control.

Through high-throughput sequencing data analysis, a total of 264259 high-quality and valid sequencing results, with an average length of 459 , were obtained from soil and groundwater bacteria samples from six groups of contaminated sites. The sequence clustering was divided with $97 \%$ consistency to obtain 3,840 OTUs. Table 1 presented the results of Unique Tags, OUT quantity and Alpha diversity index in the soil and groundwater bacteria samples from six pollution sites. The coverage of sample libraries in each group was higher than $99.5 \%$, and the main distribution was between $99.66 \%$ and $99.72 \%$. The gene sequences in the bacterial samples had high detection probability, and the sequencing results could well reflect the real situation of the bacteria community in the contaminated site soil and groundwater.

Table 1. High-throughput sequencing results of the bacteria in groundwater and soil

\begin{tabular}{c|c|c|c|c|c}
\hline Treatments & OTUs & Unique tags & Chao1 & Shannon & Coverage \\
\hline CK & 681 & 33532 & 977.39 & 5.05 & 0.9971 \\
SD1 & 994 & 39555 & 1216.46 & 5.69 & 0.9966 \\
SD2 & 1109 & 39657 & 1318.59 & 5.19 & 0.9968 \\
GW1 & 1279 & 56658 & 1578.24 & 6.30 & 0.9968 \\
GW2 & 842 & 47606 & 1093.45 & 5.57 & 0.9972 \\
GW3 & 893 & 47288 & 1173.23 & 5.84 & 0.9971 \\
\hline
\end{tabular}

CK group represented the blank control group for bacteria sequencing; SD1 and SD2 represented the control group with soil depth of $4 \mathrm{~m}$ and $7 \mathrm{~m}$ respectively; GW1, GW2, and GW3 represented the bacteria control group in groundwater when the contaminant content was low, medium, and high, respectively

Chao1 index is used to predict the total number of OUT (microbial species) in the sample, which reflects the abundance of bacterial population to some extent. The higher the Chaol index is, the higher the richness of the bacterial community will be. As shown in Table 1, the bacterial abundance in soil showed little difference at different depths, i.e. different pollution levels, but there were significant differences in the bacterial abundance in groundwater at different pollution levels $(\mathrm{p}<0.05)$. Under the condition of medium pollutant content and high pollutant content, the bacterial abundance in groundwater was significantly reduced $(\mathrm{p}<0.05)$, while the bacterial abundance in soil was not different with the influence of soil pollutant concentration. It suggested that the bacteria in groundwater were more sensitive to the change between the pollutant concentration of GW1-GW2, and the bacteria abundance would be greatly changed.

The Shannon index reflects the degree of $\alpha$-diversity of species in the microbial community, and the higher the value of Shannon index is, the higher the degree of diversity will be. From Table 1, it could be seen that different depths, i.e. different pollution degrees, had smaller differences in the diversity of bacteria in soil, but there 
were also significant differences in the diversity of bacteria in groundwater with different pollution levels $(\mathrm{p}<0.05)$. Under the condition of medium pollutant content, the bacterial diversity in groundwater was significantly reduced $(\mathrm{p}<0.05)$, while the bacterial diversity in soil did not change significantly with the influence of soil pollutant concentration. This also indicated that the concentration of pollutants between GW1GW2 will have a huge impact on the diversity of bacteria. The bacteria in groundwater were more sensitive to the change of pollutant concentration, which was consistent with the change of the abundance of the bacteria community mentioned above.

The results of the change of bacterial community abundance showed that Bacillus can promote the bio-abundance of Bacillus in medium level pollution (see Fig. 2 for details). In addition, compared with the community abundance of the control group, Comamonadaceae and Methylophilaceae had higher community abundance at this stage, while in the stage of pollution, the community abundance was low and exhibited extreme sensitivity, which could be used as the signature microorganism at this stage. Rhizobium and Pseudomonas were found in highly contaminated soil as two types of high community abundance, and the community abundance was reduced in both pollution-free and low-pollution states. Lysobacter, as a marker of low pollution in groundwater, was low in other pollution levels. Hydrogenophaga and Bacillus could be used as indicators of moderate groundwater pollution, while Brevundimonas could be used as markers of bacteria in highly polluted groundwater. In this way, Brevundimonas, Rhizobium and Pseudomonas might also be pollutant degradingbacteria.

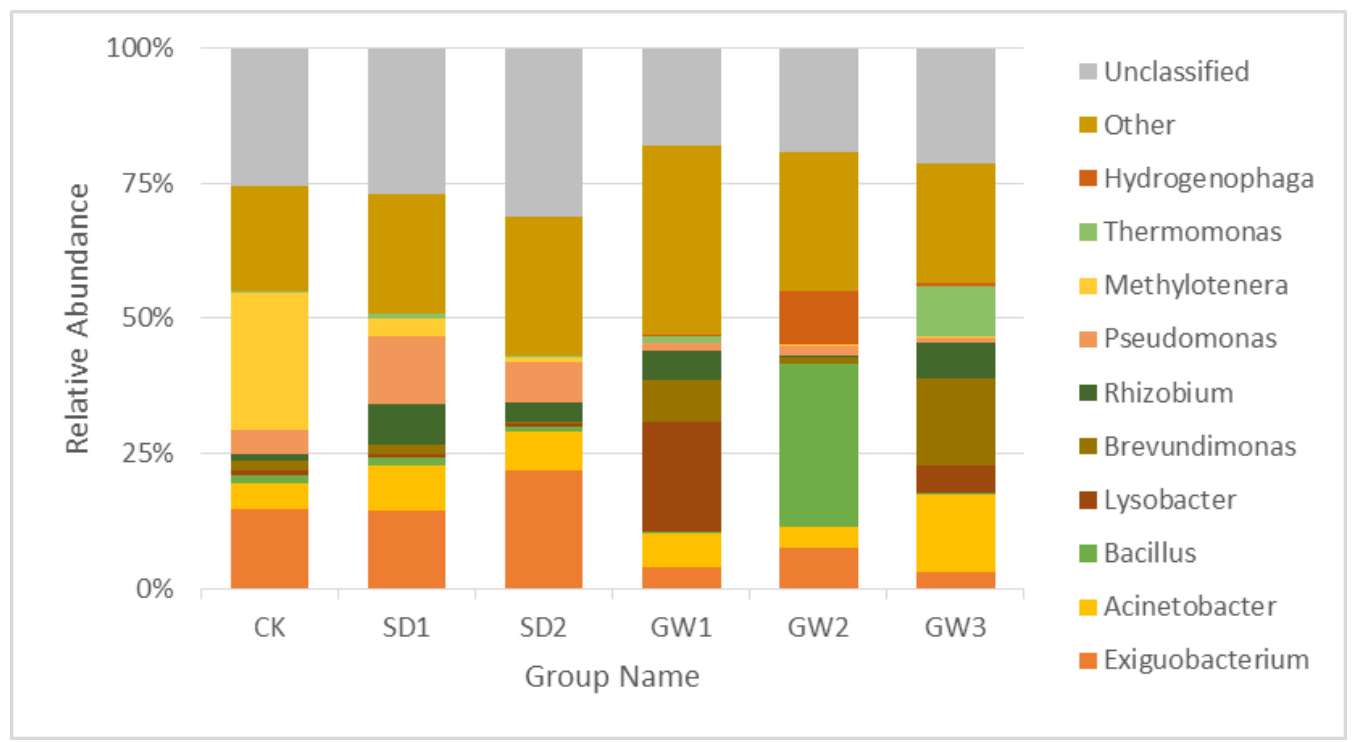

Figure 2. Bacterial species abundance of soil and groundwater

Through the primary ingredient analysis by OTU of bacteria in the control group and different environmental media, it could be seen (Fig. 3) that the cumulative contribution of bacteria in the first and second components of the six groups was $35.3 \%$ and $21.5 \%$, respectively. The results showed that in different pollution levels and environmental media, the medium groundwater environment had the greatest impact on the diversity of communities, while the groundwater with high and low pollution levels did not show 
apparent changes in the overall structure. For both soil and control groups, the diversity of their communities was generally similar.

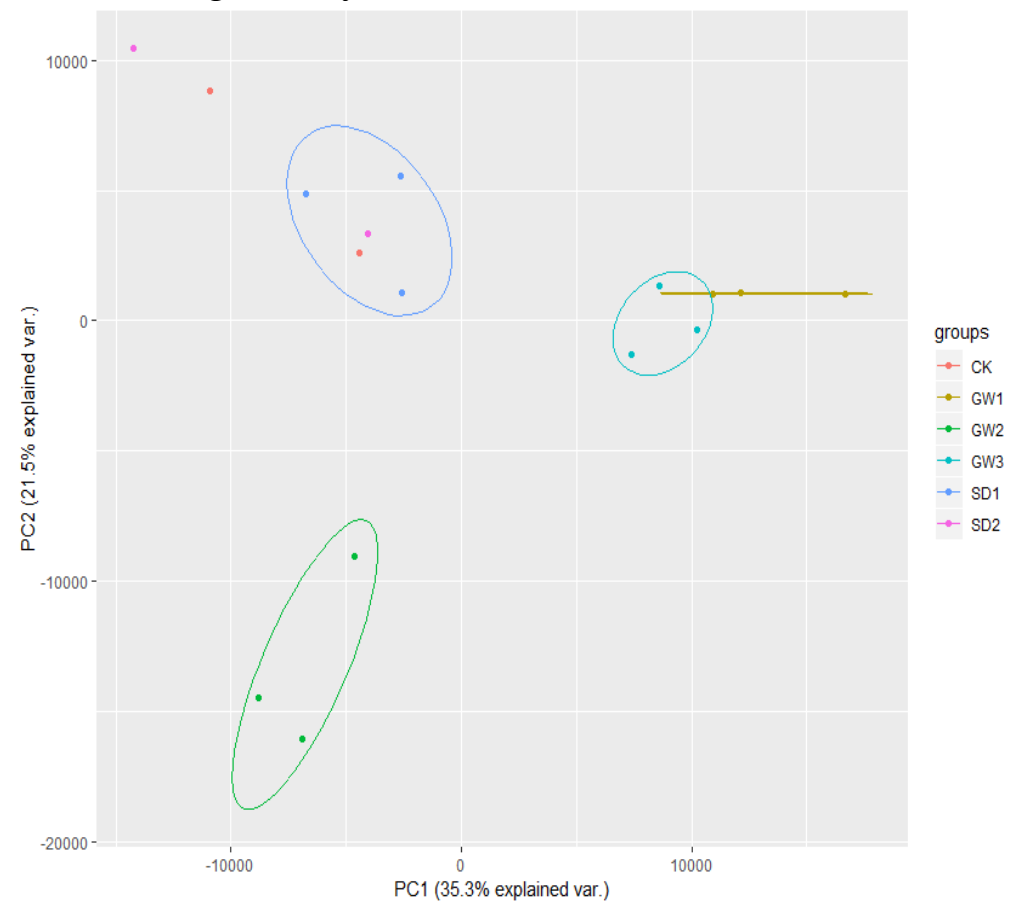

Figure 3. OTU principal component analysis for the bacteria abundance in the control group and different environmental media

Bacterial community of groundwater samples was analyzed by analyzing the main components of the group of bacteria OTU in the control group and the groundwater sample group under different pollution conditions. It could be seen (Fig. 2) that the accumulative contribution of bacteria in the first and second main components of the four samples was $42.8 \%$ and $21.4 \%$, respectively. The control group and the contaminated groundwater sample group were separately clustered and far away, indicating that the pollution would lead to large differences in the diversity of bacteria in the groundwater environment. However, in the groundwater with different pollution levels, the bacteria group with medium pollution level was significantly separated from the bacteria group with high pollution level and the bacteria group with low pollution level in the first principal component. The distance was relatively far, indicating that the groundwater with medium pollution level would produce large and significant differences in the diversity of bacteria. Compared with the bacteria of high pollution level, the bacteria of low pollution level in groundwater were divided in the second principal component and did not coincide in the $95 \%$ confidence interval, indicating that the diversity of the bacterial community was also different between high and low pollution levels.

\section{Analysis of fungi diversity in site soil and groundwater}

A total of 103254 high quality and valid sequencing results, with an average length of 357, were obtained from soil and groundwater fungi samples in 6 groups by highthroughput sequencing data analysis. The sequence clustering was divided with $97 \%$ consistency, and 652 OTUs were obtained. Table 2 showed the results of Unique Tags, 
OUT quantity and Alpha diversity index in the soil and groundwater fungi samples of six pollution sites. The sample library coverage of each group was as high as $99.9 \%$, and the main distribution was between $99.95 \%$ and $99.97 \%$. The gene sequences in the fungi samples had a high probability of detection, and the sequencing results could well reflect the real situation of the fungi community in the contaminated site soil and groundwater.

Chao1 index is used to predict the total number of OUT (microbial species) in the sample, which reflects the fungi population abundance to a certain extent. The higher the Chao1 index is, the higher the richness of the fungi community will be. It could be seen from Table 2 that the fungi abundance in soil was not significantly different with different depths, namely different pollution levels, and the fungi abundance in groundwater with different pollution levels was also similar.

Shannon index reflects the degree of diversity of species in the community of fungi, and the higher the value of Shannon index is, the higher the degree of diversity will be. It could be seen from Table 2 that unless there was a significant difference in the diversity of fungi community in transenvironmental media, the diversity of fungi community was the same in soil and groundwater with different pollution levels. The extent of the contamination has caused a small change in the growth of the fungi population. Therefore, it could be seen that the changes of fungi abundance and diversity in contaminated soil and groundwater environments were not obvious in the large structure level, which further required investigation.

Table 2. High-throughput sequencing results of the fungi of in groundwater and soil

\begin{tabular}{c|c|c|c|c|c}
\hline Sample name & OTUs & Unique tags & Chao1 & Shannon & Coverage \\
\hline CK & 176 & 17728 & 204.07 & 2.69 & 0.9997 \\
SD1 & 190 & 15245 & 240.89 & 2.13 & 0.9996 \\
SD2 & 201 & 19651 & 224.80 & 3.71 & 0.9997 \\
GW1 & 216 & 14908 & 258.73 & 4.08 & 0.9995 \\
GW2 & 169 & 19009 & 227.81 & 3.12 & 0.9997 \\
GW3 & 199 & 16713 & 235.30 & 3.55 & 0.9996 \\
\hline
\end{tabular}

CK group represented the blank control group for bacteria sequencing; SD1 and SD2 represented the control group with soil depth of $4 \mathrm{~m}$ and $7 \mathrm{~m}$ bacteria; GW1, GW2, and GW3 represented the bacteria control group in groundwater when the contaminant content was low, medium, and high, respectively

Through high-throughput sequencing analysis of fungi samples in soil and groundwater, 652 OTUs belonged to 6 phyla, 16 classes, 35 orders, 67 families and 94 genera respectively. Alternaria of Pleosporaceae followed the above community abundance characteristics in groundwater, showing that the community abundance increased with the increase of pollution concentration in the environment. In addition, compared with each group, the community richness of the Penicillium in the highly polluted soil environment was higher, while that of the other environments was lower, which might indicate the characteristic expression of the Penicillium in the soil environmental pollution at this stage. However, Fusarium had a much higher abundance in low-concentration groundwater environment than that under other conditions. The abundance of Gibberella and Cladosporium in a moderately polluted groundwater environment was much higher than that under other conditions. The abundance of Lasiodiplodia in the groundwater environment with a high concentration of pollution 
was much higher than that under other conditions (Fig. 4). All illustrate that these four strains could be as each phase of the identification of strains of bacteria and sign.

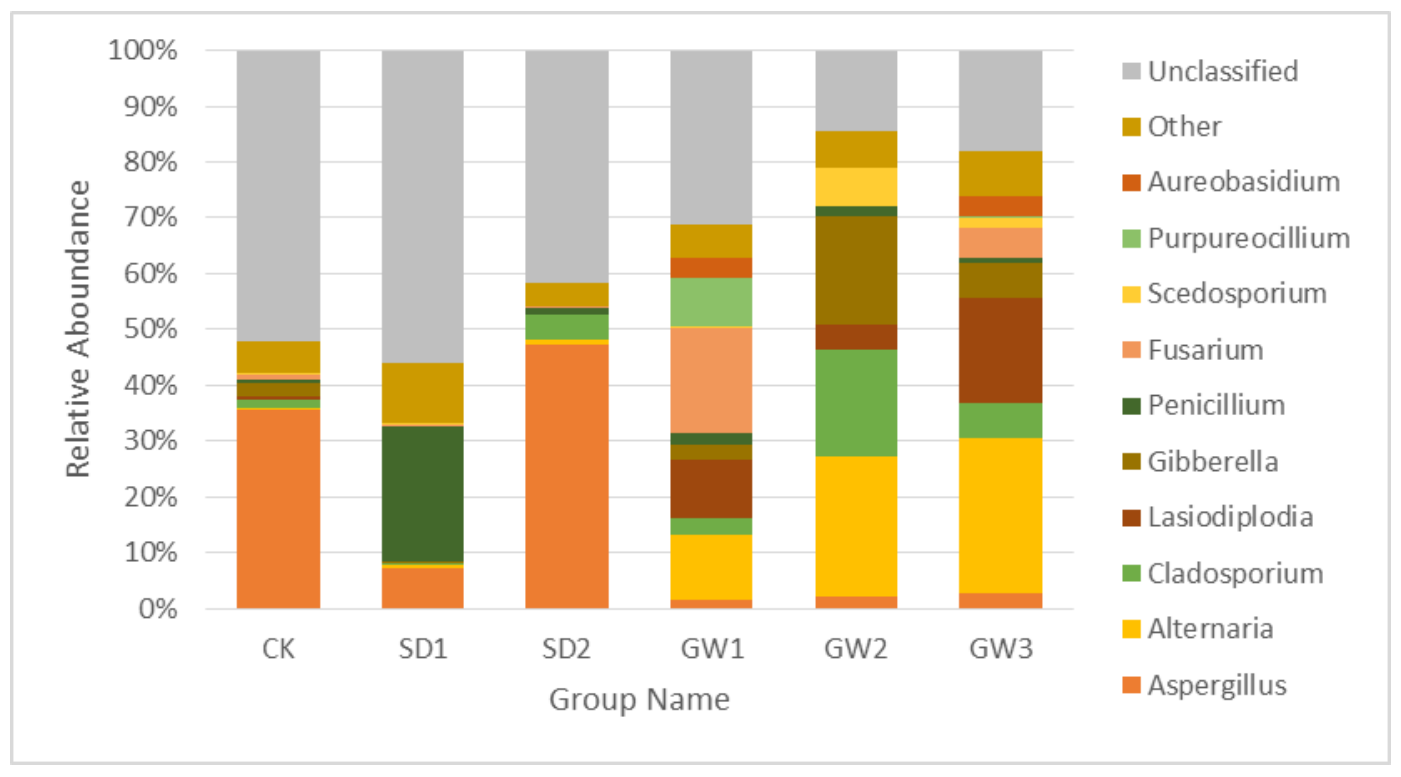

Figure 4. Fungus abundance of soil and groundwater

By principal component analysis of the OTU of the fungus in the control group and different environmental media, it could be seen (Fig. 5) that the accumulative contributions of fungi in the first and second main components of the six groups were $32.6 \%$ and $19.3 \%$, respectively. The separation of the two groups was relatively distant, indicating that the pollutant would lead to the change of community diversity. Compared with the groundwater environment, the shallow soil environment with high pollution had more changes in the first principal component. The community diversity under three kinds of groundwater environment was not much different.

\section{Discussion}

Considering the diversity of microbial community structure and function, there is a huge potential value in investigating and assessing the risk of organic contaminated sites such as chlorinated hydrocarbons. In recent years, organizations and researchers at home and abroad have carried out a large number of related experiments and achieved remarkable results. Current experimental studies have found that the structure and functional diversity of microbial communities in contaminated sites are highly correlated with the degree of contamination in contaminated sites. For highly contaminated soil and groundwater environment, the microbial community structure will be destroyed by pollutants, and the diversity of microbial communities will decline significantly compared with natural soil (Goh et al., 1997; Fu, 2018). This study has shown that the composition and structure of the soil and groundwater with different pollution levels were significantly different. And no matter in the soil or the water environment, the higher the concentration of pollutants are, the lower the microbial community abundance is, which was consistent with the results of Fantroussi et al. (1999), Banerjee et al. (1997) and Teng et al. (2004). The site was heavily contaminated 
with chlorinated hydrocarbons and the structural and functional diversity of the internal microbial population changed significantly. There are several reasons for this phenomenon. On the one hand, high concentrations of pollutants, such as chlorinated hydrocarbons, benzenes, heavy metals, etc., have poisoned and inhibited the physiological activities of microorganisms in the environment, resulting in changes in the community structure of microorganisms. Most of the sensitive microorganisms gradually died and the reproductive ability declined under the external high pollution environment, while other microorganisms with higher anti-pollution capabilities survived in the soil and groundwater. Eventually, the diversity of microbial in the environment declined. On the other hand, pollutants such as chlorinated hydrocarbons would gradually accumulate in microorganisms and some protozoa over time, which had a chronic toxic effect (Shi et al., 2010). Under the effect of bioaccumulation, the mortality rate of microorganisms and protozoa gradually increased and the reproduction rate gradually decreased, further causing changes in community structure and composition and aggravating the attenuation process of biodiversity.

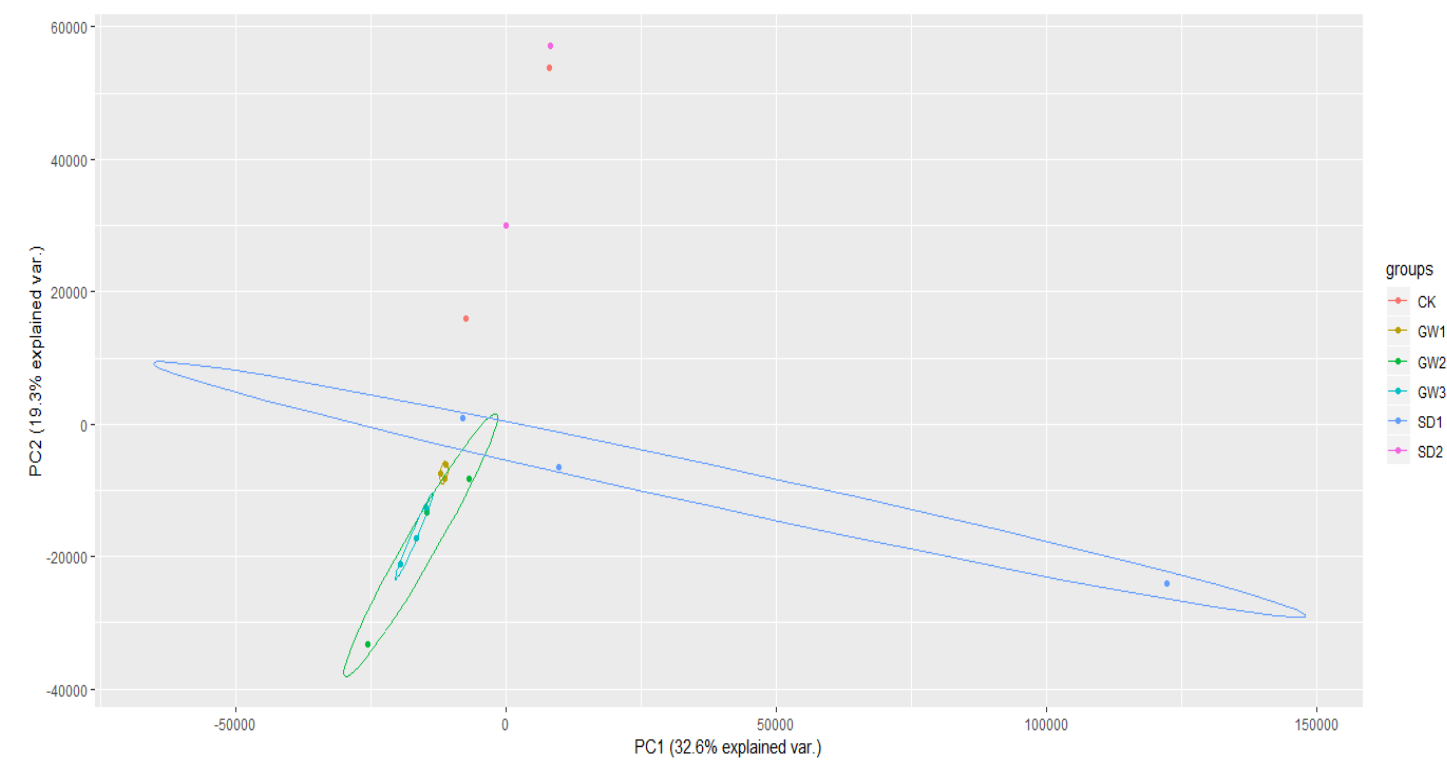

Figure 5. OTU principal component analysis for the fungus abundance in the control group and different environmental media

Besides, due to the residual effect of chlorinated hydrocarbon pollutants, there is a huge security threat to the sustainable development of human society and the balance of the natural environment. Through a large number of experimental studies, researchers and environmentalists at home and abroad have discovered that they can use the characteristics of microbial community structure and functional diversity to degrade chlorinated hydrocarbon organic pollution. As early as in the 1990s, Walter Reineke (Fritz et al., 1992) has studied the degradation of Pseudomonas to chlorobenzene and the toxicity of chlorobenzene to Pseudomonas. Shirley F (Nishino et al., 1987) reported that Pseudomonas putida could survive in soil and groundwater contaminated by chlorobenzene for a long time. This might be because that Pseudomonas was highly resistant to chlorobenzene toxicity, and they could use chlorobenzene as a carbon source for their own growth and reproduction, and absorb and degrade it to maintain their own 
life activities (Song et al., 2007). In addition, Krausova et al. (2006) also found that a microbial community consisting of Acinetobacter and Pseudomonas can degrade methane in river water to more than $80 \%$ within six days. The experiment finds that the main types of anti-pollution bacteria in the groundwater were Brevunmdimonas, Pseudomonas and Rhizobium Frank. They were abundant in groundwater and could effectively degrade chlorinated hydrocarbon pollutants in water, which were consistent with the results of Walter Reineke (Fritz et al., 1992), Shirley F (Nishino et al., 1987), Krausova (2006) and Wright et al. (2017).

The existence of microorganisms is not only positive for remediation and limiting the expansion of pollution, but also the main influencing factor for determining the structure and functional diversity of microbial populations in contaminated sites. Under the original natural environmental conditions, the microbial community structure and functional diversity in soil and groundwater depend on the interaction between various microorganisms and the external environment. With the infiltration of external pollutants, the structure and diversity change. Some sensitive microorganisms tend to be inferior, and the remaining microorganisms with higher tolerance and ability to degrade external pollutants gradually take advantage, even by using pollutants as energy and nutrient sources to enhance the abundance of their communities. Wright et al. (2017) found that in the presence of methylene chloride and trichloroethylene, the relative abundance of Pseudomonas was significantly increased, and different levels of methylene chloride contamination in the site would cause different changes in microbial community structure. Taseli and Gokcay (2005) tested Penicillium's ability to degrade chlorinated compounds, and found it could remove not only the pentachlorophenol, but also the 2-chlorophenol and trichloroacetic acid, which all present great biodegrability. In addition to the bacteria, Fungus also show up their extraordinary ability in degrading chlorinated and polycyclic aromatic hydrocarbons, which even can be enhanced by oxidation (Marco-Urrea et al., 2015). The experimental analysis found that Penicillium was the dominant flora in the soil and had high abundance, while Phylum Firmicutes was a sensitive population, which was greatly affected by chlorinated hydrocarbon pollutants, and the result was consistent with the results of Carvalho M. B. et al. (2009). Additionally, the advantaged Fungi in the environment was Aspergillu, while Fusarium spp. was a more sensitive population.

But there also exist some limitations in this study. Through the high-throughput sequencing technology, we discovered the dominated and sensitive species in the chlorinated hydrocarbon contaminated site, which could provide some insights for assessment and remediation of organochlorine contaminated sites. However, limited by time and energy, more in-depth research is expected to be conducted on the selected species to explore its degradation mechanism and sensitivity threshold. In addition, the collection of samples from multiple regions can bring more general results and avoid the differences in community distribution levels caused by regional differences. In spite of that, this study still has its profound practical significance, and plays a connecting role in the future restoration of organochlorine contaminated sites.

\section{Conclusion}

The project analyzed the structural and functional diversity of microbial communities in chlorinated hydrocarbon contaminated sites, and selected microbial flora that could maintain its own advantages in the chlorinated hydrocarbon pollution environment and 
have certain ability to degrade pollutants, which not only proved the analysis of the microbial indicators of the site can be used to assess the pollution degree and risk of the contaminated site, and provided a scientific and specific theoretical basis for the development and application of in situ bioremediation technology for chlorinated hydrocarbon contaminated sites. While in order to solid the results and make the conclusion taken into practice, further research will be necessary to select and cultivate microbial ability to degrade pollutants.

Acknowledgements. This work is supported by the Open Project of Jiangsu Key Laboratory of Environmental Engineering (ZX2016011), the Open Research Fund of Jiangsu Province Key Laboratory of Environmental Engineering (ZX2016012) and the Research project for environmental protection of Jiangsu Province (2017001). We thank Kai Sun at Southeast University for the constructive comments of this paper.

\section{REFERENCES}

[1] Banerjee, M. R., Burton, D. L., Depoe, S. (1997): Impact of sewage sludge application on soil biological characteristics. - Agriculture Ecosystems \& Environment 66(3): 241-249.

[2] Carvalho, M. B., Martins, I., Leitão, M. C., Garcia, H., Rodrigues, C., Romão, V. S., McLellan, I., Hursthouse, A., Pereira, C. S. (2009): Screening pentachlorophenol degradation ability by environmental fungal strains belonging to the phyla Ascomycota and Zygomycota. - Journal of Industrial Microbiology and Biotechnology 36(10): 12491256.

[3] El Fantroussi, S., Verschuere, L., Verstraete, W., Top, E. M. (1999): Effect of phenylurea herbicides on soil microbial communities estimated by analysis of 16S rRNA gene fingerprints and community-level physiological profiles. - Applied and Environmental Microbiology 65(3): 982-8.

[4] Fritz, H., Reineke, W., Schmidt, E. (1992): Toxicity of chlorobenzene on Pseudomonas sp. strain RHO1, a chlorobenzene-degrading strain. - Biodegradation 2: 165-170.

[5] Fu, W. Y. (2018): Chemical Oxidation Remediation and Microbial Community Diversity Research of PAHs Contaminated Soil. - East China Normal University, Shanghai.

[6] Gao, H. P. (2014): Bioavailability Assessment and Biodegradation-Promoting Method for Persistent Organic Pollutants in Soil. - Dalian University of Technology, Liaoning.

[7] Goh, T. B., Banerjee, M. R. (1997): Vesicular arbuscular mycorrhizae -mediated uptake and translocation of $\mathrm{P}$ and $\mathrm{Zn}$ by wheat in calcareous soil. - Pedosphere 7: 317-324.

[8] Krausova, V. I., Robb, F. T., González, J. M. (2006): Biodegradation of dichloromethane in an estuarine environment. - Hydrobiologia 559: 77-83.

[9] Li, Y., Xu, X. X. (2019): Research progress of high-throughput sequencing technology. China Medical Engineering 3: 32-37.

[10] Liang, J. G., Liu, P. C., Zhang, X. J. (2018): Impact of genetically modified crops on rhizosphere bacterial community structure based on 16S rDNA high-throughput sequencing. - Jiangsu Agricultural Science 46: 5-8.

[11] Luo, Q., Huang, B. L., Tang, Z. X., Lai, L. M., Wei, W., Zheng, Y. R. (2013): Carbon source utilization of microbes in saline soil of three vegetation types in Xinjiang, China. Chinese Journal of Applied Environmental Biology 19: 96-104.

[12] Marco-Urrea, E., Garcia-Romera, I., Aranda, E. (2015): Potential of non-ligninolytic fungi in bioremediation of chlorinated and polycyclic aromatic hydrocarbons. - New Biotechnology. 32(6): 620-628.

[13] Nie, X. J. (2013): Preliminary Genomic Study of Two Non-Mondel Plants: Wheat (Triticum aesticum L) and Crofton Weed (Ageratina adenophora) through HighThroughput Sequencing Technology. - Northwest A\&F University, Yangling. 
[14] Nishino, S. F., Spain, J. C. (1987): Degradation of 1,4-dichlorobenzene by a Pseudomonas sp. - Applied and Environmental Microbiology 53: 1010-1019.

[15] Qiu, J., Hou, Y. L., Xu, L. L., Qian, Y, Ding, X. (2019): High-throughput sequencing analysis of rhizosphere soil bacteria diversity in different mulberry varieties. - Journal of Southern Agriculture 3: 585-592.

[16] Rosselli, R., Romoli, O., Vitulo, N., Vezzi, A., Campanaro, S., Pascale, F. D., Schiavon, R., Tiarca, M., Poletto, F., Concheri, G., Valle, G., Squartini, A. (2016): Direct 16S rRNA-seq from bacterial communities: a PCR-independent approach to simultaneously assess microbial diversity and functional activity potential of each taxon. - Scientific Reports 6: 32165.

[17] Shi, H. L., Su, Y., Xu, L., Wang, Y. (2018): The industry status and development trend of high-throughput sequencing. - Biotechnology 3: 6-12.

[18] Shi, Z., Zhang, D. W., Huang, X. P., Wang, F. (2010): Bioaccumulation of organochlorine pesticides in organisms and sediments in the western coastal waters of Guangdong province. - Journal of Tropical Oceanography 29: 114-119.

[19] Song, L., Wang, H., Jiang, J., Gao, J. S., Shi, H. C. (2007): Isolation, identification of 1,2,4-trichlorobenzene-degrading strain Pseudomonas nitroreducens J5-1 and cloning of chlorocatechol 1,2-dioxygenase gene. - Environmental Science 8: 1878-1881.

[20] Song, X., Lin, N., Yin, P. H. (2015): Contaminated site remediation industry in China: current state and future trends. - Soils 47: 1-7.

[21] Su, G. J., Huang, L. Y., Lu, H. J., Li, Q. Q. (2015): Preparation and application of nickelcobalt-iron ternary composite nano-metal oxide: China, Research Center for EcoEnvironmental Sciences. - Chinese Academy of Sciences, Beijing.

[22] Tan, L. L., Li, J. Z., Wu, H. X. (2017): Evaluation of soil and groundwater pollution in typical organic chemical plant. - Environment \& Development 29: 68-69.

[23] Taseli, B. K., Gokcay, C. F. (2005): Degradation of chlorinated compounds by Penicillium camemberti in batch and up-flow column reactors. - Process Biochemistry 40(2): 917-923.

[24] Teng, Y., Huang, C. Y., Luo, Y. M., Long, J., Yao, H. Y. (2004): Microbial activities and functional diversity of community in soils polluted with $\mathrm{Pb}-\mathrm{Zn}-\mathrm{Ag}$ mine tailings. - Acta Pedologica Sinica 41(1): 113-119.

[25] Wang, H. P. (2018): Investigation and remediation of chlorinated hydrocarbon aromatics complex site. - China Resources Comprehensive Utilization 36: 141-142.

[26] Wright, J., Kirchner, V., Bernard, W., Ulrich, N., McLimans, C., Campa, M. F., Hazen, T., Macbeth, T., Marabello, D., McDermott, J., Mackelprang, R., Roth, K., Lamendella, R. (2017): Bacterial community dynamics in dichloromethane-contaminated groundwater undergoing natural attenuation. - Frontiers in Microbiology 8. DOI: 10.3389/fmicb.2017.02300.

[27] Wu, J. N., Liu, Y. X., Zhou, X., Wang, T. Y., Gao, Q., Gao, Y. H., Liu, S. X. (2018): Effects of long-term different fertilization on soil fungal communities in black soil based on the Illumina MiSeq platform. - Acta Microbiologica Sinica 58: 1658-1671.

[28] Zhang, X., Song, S. S., Huang, Y. P., Jia, Z. H., Huang, Y. Y., Song, C. (2018): Analysis of bacterial diversity in cultivated soils in middle and southern Hebei Province based on high-throughput sequencing. - North China Agricultural Journal 33: 196-203. 